\title{
Histone deacetylases 1 and 2 cooperate in regulating BRCA1, CHK1, and RAD51 expression in acute myeloid leukemia cells
}

\author{
Jianyun Zhao ${ }^{1,4,5, *}$, Chengzhi Xie ${ }^{2,3, *}$, Holly Edwards ${ }^{2,3}$, Guan Wang1, Jeffrey W. \\ Taub $^{3,4,5}$, Yubin $\mathbf{G e}^{1,2,3,4}$ \\ ${ }^{1}$ National Engineering Laboratory for AIDS Vaccine and Key Laboratory for Molecular Enzymology and Engineering, The \\ Ministry of Education, School of Life Sciences, Jilin University, Changchun, P. R. China \\ ${ }^{2}$ Department of Oncology, Wayne State University School of Medicine, Detroit, MI, USA \\ ${ }^{3}$ Molecular Therapeutics Program, Barbara Ann Karmanos Cancer Institute, Wayne State University School of Medicine, \\ Detroit, MI, USA \\ ${ }^{4}$ Department of Pediatrics, Wayne State University School of Medicine, Detroit, MI, USA \\ ${ }^{5}$ Division of Pediatric Hematology/Oncology, Children's Hospital of Michigan, Detroit, MI, USA \\ *These authors are contributed equally to this work \\ Correspondence to: Yubin Ge, email: gey@karmanos.org \\ Jeffrey W. Taub, email: jtaub@med.wayne.edu
}

Keywords: HDAC, BRCA1, CHK1, RAD51, acute myeloid leukemia

Received: October 19, $2016 \quad$ Accepted: December 13, 2016

Published: December 21, 2016

\section{ABSTRACT}

Resistance to chemotherapy and a high relapse rate highlight the importance of finding new therapeutic options for the treatment of acute myeloid leukemia (AML). Histone deacetylase (HDAC) inhibitors (HDACIs) are a promising class of drugs for the treatment of AML. HDACIs have limited single-agent clinical activities, but when combined with conventional or investigational drugs they have demonstrated favorable outcomes. Previous studies have shown that decreasing expression of important DNA damage repair proteins enhances standard chemotherapy drugs. In our recent studies, the pan-HDACI panobinostat has been shown to enhance conventional chemotherapy drugs cytarabine and daunorubicin in AML cells by decreasing the expression of BRCA1, CHK1, and RAD51. In this study, we utilized class- and isoform-specific HDACIs and shRNA knockdown of individual HDACs to determine which HDACs are responsible for decreased expression of BRCA1, CHK1, and RAD51 following pan-HDACI treatment in AML cells. We found that inhibition of both HDAC1 and HDAC2 was necessary to decrease the expression of BRCA1, CHK1, and RAD51, enhance cytarabine- or daunorubicin-induced DNA damage and apoptosis, and abrogate cytarabine- or daunorubicin-induced cell cycle checkpoint activation in AML cells. These findings may aid in the development of rationally designed drug combinations for the treatment of AML.

\section{INTRODUCTION}

The standard treatment for most acute myeloid leukemia (AML) patients, consisting of cytarabine (ara-C) and an anthracycline [e.g., daunorubicin (DNR)], has been used for the past four decades. Though a high percentage of patients respond to induction therapy, a majority relapse [1]. AML is typically diagnosed in elderly individuals (median age 60-65 years); this population has higher rates of therapy-related relapse and decreased efficacy $[2,3]$. Therefore, more effective therapies are urgently needed to improve treatment outcome of AML patients.

Histone deacetylase (HDAC) inhibitors (HDACIs) are a class of antileukemic agents of particular promise due to their effects on cell differentiation, cell cycle arrest, and apoptosis in human leukemic cells, but less so in normal cells $[1,4,5]$. However, the pleiotropic spectrum of pan-HDACIs makes it difficult to investigate the specific function of individual HDACs which contribute to the antileukemic effect. Although HDACIs have limited single-agent clinical activities [6-10], they have 
demonstrated promising results when combined with conventional and investigational drugs for the treatment of AML [11-13]. We previously demonstrated that panobinostat (a pan-HDACI) suppressed the expression of BRCA1, CHK1, and RAD51 which play critical roles in the DNA damage response (DDR), leading to induction of DNA DSBs and apoptosis, and abrogation of the activation of the cell cycle checkpoints induced by ara-C or DNR in AML cells [14]. However, it is still unknown which HDAC isoforms play a key role in regulating the expression of these proteins.

In this study, we demonstrate that together HDAC1 and HDAC2 activities are responsible for the decreased expression of BRCA1, CHK1, and RAD51 following class I- or pan-HDACI treatment in AML cells. By treating THP-1 and OCI-AML3 cell lines with class-, subclass-, and isoform-selective HDACIs, we found that simultaneous inhibition of HDAC1 and HDAC2 caused decreased expression of BRCA1, CHK1, and RAD51. Inhibition of HDAC1 and HDAC2 enhanced DNA DSBs and apoptosis induced by ara-C or DNR. Furthermore, it abrogated the activation of the $\mathrm{S}$ and $\mathrm{G} 2$ cell cycle checkpoints induced by ara-C or DNR in AML cell lines. These findings provide a better therapeutic strategy for the development of new HDACIs for the treatment of AML.

\section{RESULTS}

\section{Class II HDACs are irrelevant with respect to the expression of $B R C A 1, C H K 1$, and $R A D 51$ in AML cells}

In our previous study, we demonstrated that the most potent pan-HDACI panobinostat induced apoptosis by suppressing the expression of DNA repair proteins BRCA1, CHK1, and RAD51 in AML cells [14]. Further, we found that inhibition of both HDACs 1 and 6 was critical for enhancing ara-C-induced apoptosis in pediatric AML cells [15]. To investigate which specific HDAC isoforms play critical roles in this process in AML cells, first we focused on Class II HDACs. We treated THP-1 and OCI-AML3 cell lines with variable concentrations of MC1568 (a Class IIa-selective HDACI) for $48 \mathrm{~h}$ and then subjected whole cell lysates to Western blotting. As shown in Figure $1 \mathrm{~A}$ and 1B, MC1568 treatment resulted in increased expression of ac-H4, but had no obvious impact on the expression of ac-tubulin. Interestingly, the expression levels of BRCA1, CHK1, and RAD51 in the AML cell lines remained largely unchanged, demonstrating that class IIa HDACs are not involved in the expression of these DDR genes (Figure 1A and 1B). Similar results were obtained when THP-1 and OCI-AML3 cells were treated with variable concentrations of Tubastatin A (a HDAC6-selective inhibitor) for $48 \mathrm{~h}$ (Figure 1C and 1D). Taken together, these results demonstrate that Class II HDACs do not disrupt BRCA1, CHK1, and RAD51 expression in AML cells.

\section{Inhibiting HDACs 1, 2, and 3 decreases the transcript and protein levels of $B R C A 1, C H K 1$, and $R A D 51$ and induces apoptosis in AML cell lines}

To determine if Class I HDACs affect the transcript and protein levels of $B R C A 1, C H K 1$, and RAD51 genes, we treated THP-1 cells with variable concentrations of MGCD0103 (a class I HDACI) for $48 \mathrm{~h}$ and then measured the enzymatic activities of HDACs 1, 2, 3, and 8 following immunoprecipitation. MGCD0103 caused significant inhibition of HDACs 1, 2, and 3 activities, but did not affect HDAC8 activity (Figure 2A). Then we measured $B R C A 1, C H K 1$, and RAD51 transcript levels by real-time RT-PCR and protein levels by Western blotting in the cell lines post MGCD0103 treatment. There was a concentration-dependent decrease of BRCA1, CHK1, and $R A D 51$ transcript and protein levels in THP-1 cells (Figure 2B and 2C). Meanwhile, MGCD0103 caused concentration-dependent increase of acetylatedhistone $\mathrm{H} 4$, while having no effect on acetylation of alpha-tubulin and total histone H4 levels (Figure 2C). Similar results were also obtained in OCI-AML3 cells (Figure 2D-2F). Interestingly, downregulation of these DDR genes by MGCD0103 treatment was accompanied by concentration-dependent induction of apoptosis in both cell lines (Figure 2F). Together, these results demonstrate that simultaneous inhibition of HDACs 1, 2, and 3 by MGCD0103 suppresses the transcript and protein expression levels of $B R C A 1, C H K 1$, and $R A D 51$ in AML cell lines.

\section{Inhibiting HDACs 1, 2, and 3 enhances the antileukemic activities of ara-C and DNR against AML cells}

To determine if inhibiting HDAC1, HDAC2, and HDAC3 enhances the antileukemic activity of ara-C or DNR, we treated THP-1 and OCI-AML3 cells with MGCD0103 and ara-C or DNR, alone or combined, for $48 \mathrm{~h}$ and then subjected the cells to Annexin V/propidium iodide (PI) staining, and flow cytometry. Consistent with panobinostat, MGCD0103 enhanced ara-C- and DNR-induced apoptosis in THP-1 and OCI-AML3 cells (Figure 3A and 3C), which was accompanied by increased DNA DSBs, as reflected by the induction of $\gamma \mathrm{H} 2 \mathrm{AX}$ (an established biomarker for DNA double-strand breaks [16], Figure $3 \mathrm{~B}$ and $3 \mathrm{D})$. In the combined treatment, we detected decreased expression of BRCA1, CHK1, and RAD51 compared to ara-C or DNR alone in both cell lines (Figure 3B and 3D). MGCD0103 treatment alone resulted in increased G0/G1 phase cells, suggesting 
$\mathrm{G} 0 / \mathrm{G} 1$ arrest and ara- $\mathrm{C}$ treatment resulted in increased $\mathrm{S}$ and $\mathrm{G} 2 / \mathrm{M}$ phase cells, suggesting $\mathrm{S}$ and $\mathrm{G} 2 / \mathrm{M}$ arrest in both cell lines. In THP-1 cells, ara-C-induced increase of $\mathrm{S}$ phase cells was reduced, while in OCI-AML3 cells ara-C-induced increase of $\mathrm{G} 2 / \mathrm{M}$ phase cells was decreased by the addition of MGCD0103 (Figure 3E and 3F). DNR treatment resulted in increased $\mathrm{G} 2 / \mathrm{M}$ phase cells, which was reduced by co-administration of MGCD0103 in both cell lines (Figure 3E and 3F). These results suggest that inhibition of HDACs 1, 2, and 3 enhances the antileukemic activity of ara-C and DNR through suppressing the expression of BRCA1, CHK1, and RAD51 in AML cells.

\section{HDACs 1 and 2 cooperate in regulating the expression of $B R C A 1, C H K 1$, and $R A D 51$ in AML cells}

To further investigate which HDAC isoforms, among HDAC1, HDAC2, and HDAC3, play important roles in regulating expression of $B R C A 1, C H K 1$, and $R A D 51$ in AML cells, we individually knocked down $H D A C 1, H D A C 2$, and $H D A C 3$ in THP-1 cells (Figure 4A, designated THP-1/HDAC1, THP-1/HDAC2, and THP-1/HDAC3, respectively; Western blot verification of knockdown was previously published [15]). Then we determined BRCA1, CHK1, and RAD51 transcript and protein levels in these cells by real-time RT-PCR and Western blotting, respectively. As show in Figure 4B and 4C, $H D A C 1, H D A C 2$ or $H D A C 3$ knockdown did not affect $B R C A 1, C H K 1$, and $R A D 51$ transcript and protein levels in
THP-1 AML cells. Next we treated THP-1/HDAC1 cells and THP-1/HDAC2 cells with variable concentrations of RGFP966, an HDAC3-selective inhibitor, for $48 \mathrm{~h}$, and then subjected the cells to Western blotting. As shown in Figure 4D and 4E, RGFP966 treatment had no impact on the expression of BRCA1, CHK1, and RAD51 compared to no drug treated control or the no drug treated NTC cells. These results suggest that simultaneous inhibition of HDAC1 and HDAC3 or HDAC2 and HDAC3 is not sufficient to reduce BRCA1, CHK1, and RAD51 expression. Then we treated THP-1 and OCI-AML3 cells with variable concentrations of FK228 (an HDAC1 and HDAC2 inhibitor) for $48 \mathrm{~h}$. Suppression of $B R C A 1$, $C H K 1$, and $R A D 51$ transcript and protein expression was detected after cells were treated with 5 nM FK228, which was accompanied by apoptosis (Figure 5). These results revealed that $\mathrm{HDAC} 1$ and $\mathrm{HDAC} 2$ cooperate in regulating $B R C A 1, C H K 1$, and $R A D 51$ transcript and protein expression in AML cells.

\section{Simultaneous inhibition of $H D A C 1$ and $H D A C 2$ enhances the antileukemic activities of ara-C and DNR against AML cells}

To determine the effects of HDAC1 and HDAC2 on the antileukemic activity of ara-C and DNR, we treated THP-1 and OCI-AML3 cells with FK228 and ara-C or DNR, alone or in combination, for $48 \mathrm{~h}$. The cells were then subjected to flow cytometry analyses for apoptosis and cell cycle progression. The results revealed that
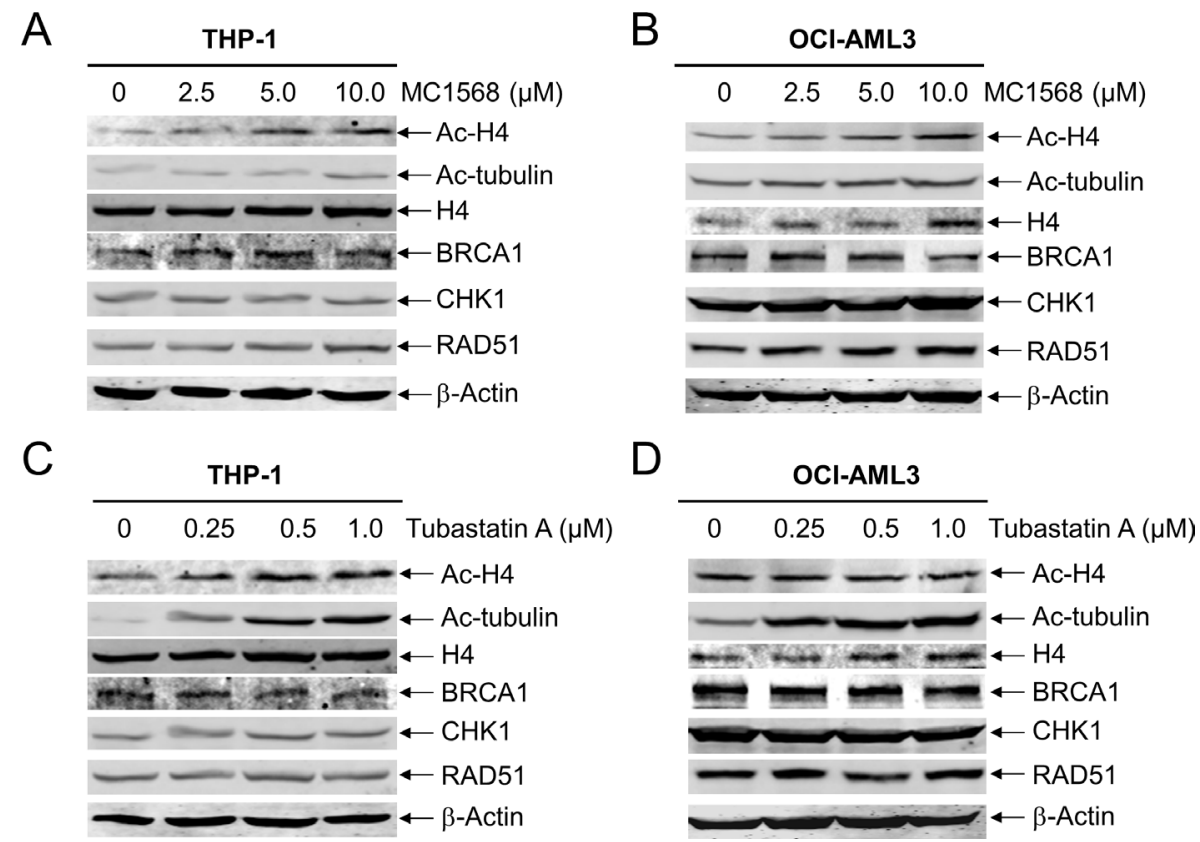

Figure 1: Inhibition of Class II HDACs has no impact on the expression of BRCA1, CHK1, and RAD51 in AML cells. (A and B) THP-1 and OCI-AML3 cells were treated with MC1568 for $48 \mathrm{~h}$, and then whole cell lysates were subjected to Western blotting and probed with the indicated antibodies. (C amd D) THP-1 and OCI-AML3 cells were treated with Tubastatin A for 48 h, and then whole cell lysates were subjected to Western blotting and probed with the indicated antibodies. 
FK228 can enhance ara-C- and DNR-induced apoptosis in THP-1 and OCI-AML3 cells (Figure 6A and 6C), accompanied by reduced expression of BRCA1, CHK1, and RAD51 compared to ara-C or DNR alone and increased DNA DSBs, as reflected by the induction of $\gamma \mathrm{H} 2 \mathrm{AX}$ (Figure 6B and 6D). FK228 treatment alone resulted in increased $\mathrm{G} 0 / \mathrm{G} 1$ phase cells in THP-1 and OCI-AML3 cells. FK228 partially abrogated ara-C-induced increase of S phase cells in THP-1 cells and decreased ara-C-induced increase of $\mathrm{G} 2 / \mathrm{M}$ phase cells in OCI-AML3 cells. DNR-induced increase of G2/M cells was reduced by co-administration with FK228 in both
A

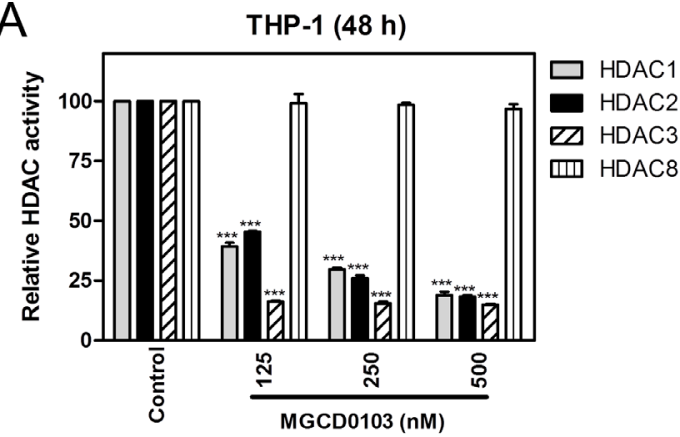

C

C

THP-1 (48 h)

$\begin{array}{llll}0 & 125 & 250 & 500 \text { MGCD0103 (nM) }\end{array}$

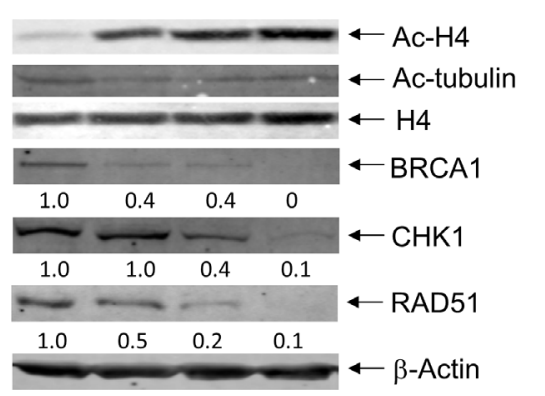

E

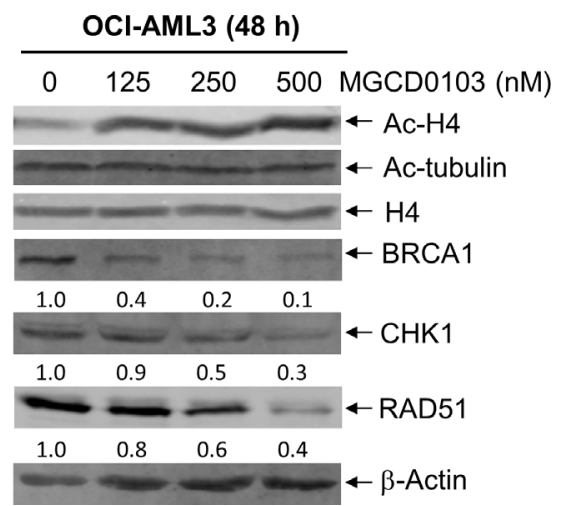

B

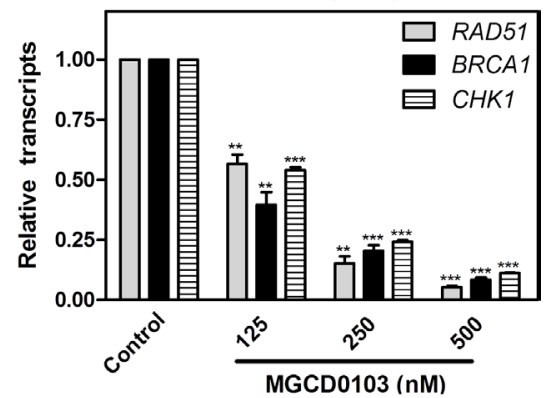

D

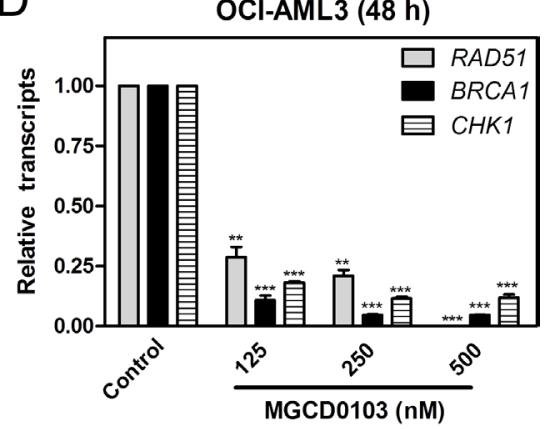

$\mathrm{F}$

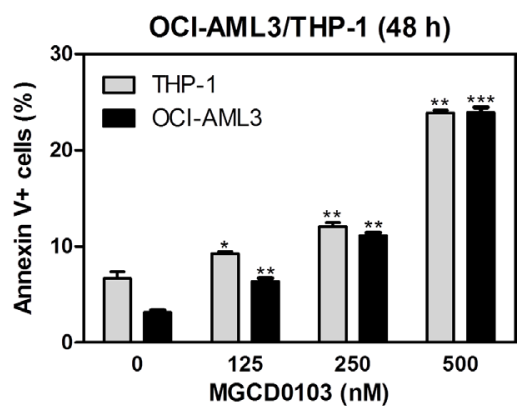

Figure 2: Inhibition of HDACs 1, 2, and 3 decreases the protein and transcript levels of BRCA1, CHK1, and RAD51, and induces apoptosis in AML cell lines. (A) THP-1 cells were treated with variable concentrations of MGCD0103 for 48 h. Protein extracts were subjected to immunoprecipitation with antibodies against class I HDACs and then class I HDAC activities were measured, as described in the Materials and Methods. (B) THP-1 cells were treated with MGCD0103 for $48 \mathrm{~h}$. Then total RNAs were isolated and gene transcript levels were determined by Real-time RT-PCR. Transcript levels were normalized to GAPDH and relative expression levels were calculated using the comparative Ct method. (C) THP-1 cells were treated with MGCD0103 for 48 h. Whole cell lysates were subjected to Western blotting and probed with the indicated antibodies. The fold changes for the densitometry measurements, normalized to $\beta$-actin and then compared to no drug treatment control, are indicated. (D) OCI-AML3 cells were treated with MGCD0103 for $48 \mathrm{~h}$, then total RNAs were isolated from treated cells and gene transcript levels were determined by Real-time RT-PCR. Transcript levels were normalized to GAPDH and relative expression levels were calculated using the comparative Ct method. (E) OCI-AML3 cells were treated with MGCD0103 for $48 \mathrm{~h}$. Whole cell lysates were subjected to Western blotting and probed with the indicated antibodies. (F) THP-1 and OCI-AML3 cells were treated with MGCD0103 for $48 \mathrm{~h}$ and then subjected to Annexin V-FITC/PI staining and flow cytometry analysis. *indicates $p<0.05$, **indicates $p<0.01$, and ***indicates $p<0.001$ (panels A, B, D \& F). 
cell lines (Figure 6E and 6F). These results are similar to those following MGCD0103 treatment, suggesting that inhibiting HDAC1 and HDAC2 has the same effect of decreasing the expression of BRCA1, CHK1, and RAD51, as inhibiting HDAC1, HDAC2 and HDAC3 together.

\section{DISSCUSSION}

In our previous study, we demonstrated that panobinostat (pan-HDACI) suppresses the expression of three critical DDR proteins, BRCA1, CHK1, and RAD51, leading to enhancement of DNA DSBs, abrogation of cell cycle checkpoints, and enhanced induction of apoptosis by ara-C or DNR in AML cells [14]. We also found that inhibition of both HDACs 1 and 6 was critical in enhancing ara-C-induced apoptosis in pediatric AML cells [15]. However, which HDAC isoforms participate in regulating the expression of BRCA1, CHK1, and RAD51 in AML cells remains unknown. Solving this problem could help us better understand the specific function of individual HDACs, which is essential for the development of new therapies, as well as for rationally designing combination therapies for the treatment of AML.

Treatment with a Class II HDACI, MC1568 or Tubastatin A, had no effect on BRCA1, CHK1, or RAD51 protein levels, demonstrating that Class II HDACs do not have an impact on the expression of these proteins. The Class I HDACI MGCD0103, which inhibits HDACs 1, 2, and 3, caused concentration-dependent decrease of both protein and
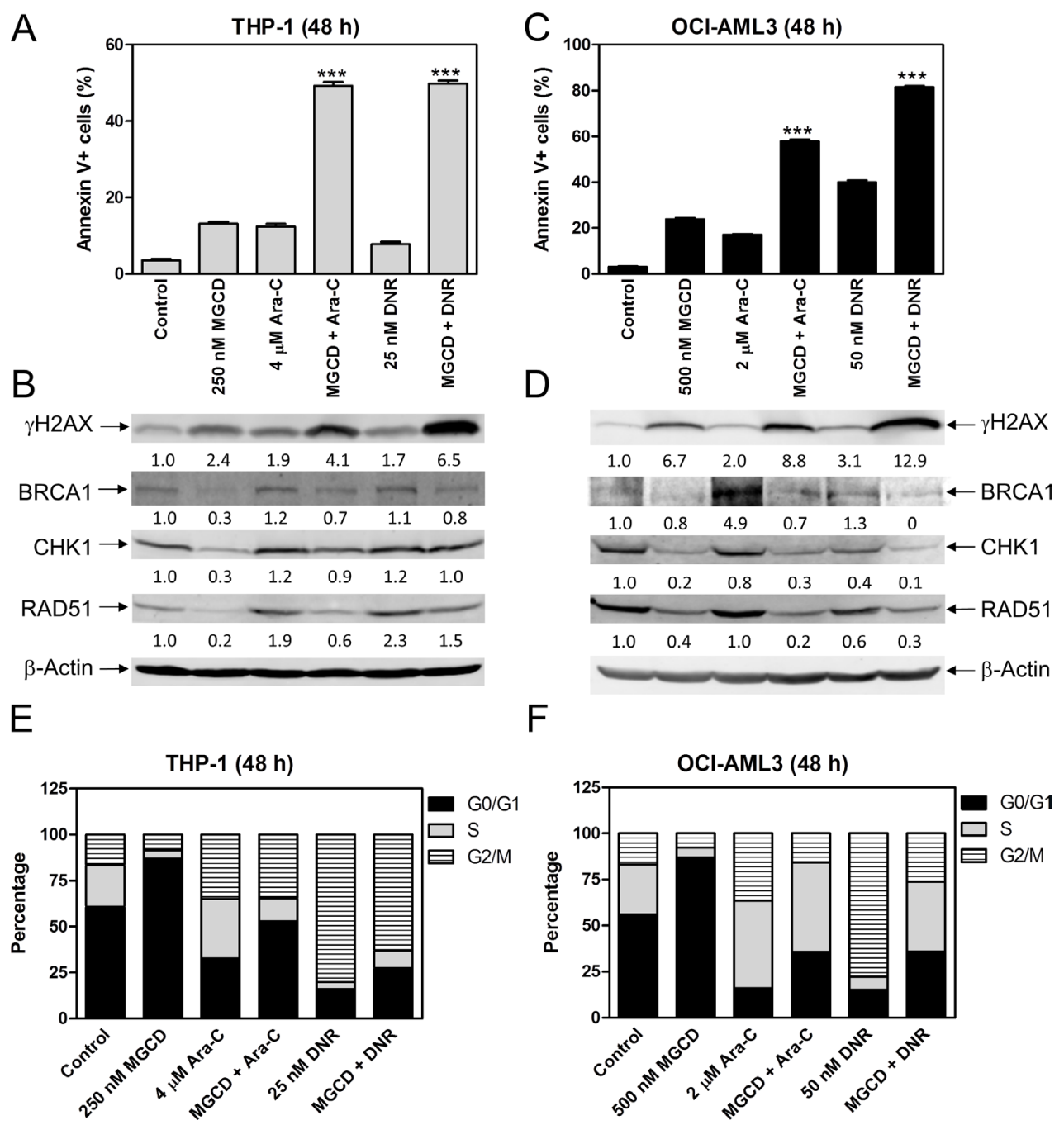

Figure 3: MGCD0103 cooperates with ara-C or DNR in inducing apoptosis and abrogates $\mathrm{S}$ and/or G2/M cell cycle checkpoint activation induced by ara-C or DNR in AML cells. (A and C) THP-1 and OCI-AML3 cells were treated with MGCD0103 and ara-C or DNR, alone or in combination, for $48 \mathrm{~h}$ and then subjected to Annexin V-FITC/PI staining and flow cytometry analyses. ***indicates $p<0.001$. (B and $\mathbf{D})$ Whole cell lysates were subjected to Western blotting and probed with the indicated antibodies. The fold changes for the densitometry measurements, normalized to $\beta$-actin and then compared to no drug treatment control, are indicated. (E and F) THP-1 and OCI-AML3 cells were treated for $48 \mathrm{~h}$ with MGCD0103 and ara-C or DNR, alone or in combination, then fixed with ethanol and stained with PI for cell cycle analysis. 
transcript levels for $B R C A 1, C H K 1$, and $R A D 51$ in THP-1 and OCI-AML3 cells. MGCD0103 enhanced DNA DSBs and apoptosis induced by ara-C or DNR, and it also abrogated $\mathrm{S}$ and/or G2/M checkpoint activation induced by ara-C or DNR in these cells (Figure 3). These results are consistent with our previous findings using panobinostat [14], suggesting that HDAC1, HDAC2, and HDAC3 participate in the regulation of BRCA1, CHK1, and RAD51 expression and play important roles in the antileukemic activities of ara-C or DNR in AML cells.

While individual knockdown of $H D A C 1, H D A C 2$ or $H D A C 3$ did not alter $B R C A 1, C H K 1$, and RAD51 expression, combined inhibition of HDAC1 and HDAC2 decreased their expression. Our results were consistent with Miller et al. who demonstrated that HDAC1 and HDAC2 promote double-strand break repair [17], though the mechanism was not determined. It has been reported that inhibition of HDAC2 causes downregulation of RAD51 in melanoma cells [18]. Thurn and colleagues have reported that inhibition or siRNA knockdown of both $H D A C 1$ and $H D A C 2$ not only resulted in decreased BRCA1 transcript levels, but also ATM transcript and protein levels as well [19]. Although we did not investigate ATM levels, HDAC1 and HDAC2 inhibition caused decreased transcript and protein levels of $B R C A 1, C H K 1$, and RAD51 in AML cells.

In summary, our study demonstrates that simultaneous inhibition of both HDAC1 and HDAC2 decreases the expression of BRCA1, CHK1, and RAD51, induces DNA DSBs and apoptosis, and abrogates cell cycle checkpoint activation induced by ara-C or DNR in AML cells. pan-HDACIs have been shown to decrease BRCA1, $C H K 1$, and $R A D 51$ expression through transcriptional regulation, of which E2F1 plays a critical role [14, 20], and post-translational mechanisms [21]. In addition, in AML cells it has been shown that inhibition of HDAC1 and HDAC2 causes upregulation of miR-182 which directly targets RAD51, resulting in decrease of RAD51 expression [22]. These studies indicate that HDAC1 and HDAC2 regulate the expression of $B R C A 1, C H K 1$, and $R A D 51$ through both transcriptional and post-translational mechanisms. Our data support combining a class I HDACI with DNA damaging agents for the treatment of AML and provide guidance for the further development of HDAC selective inhibitors.
A

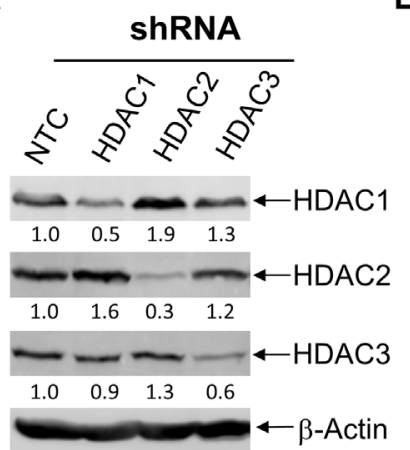

D

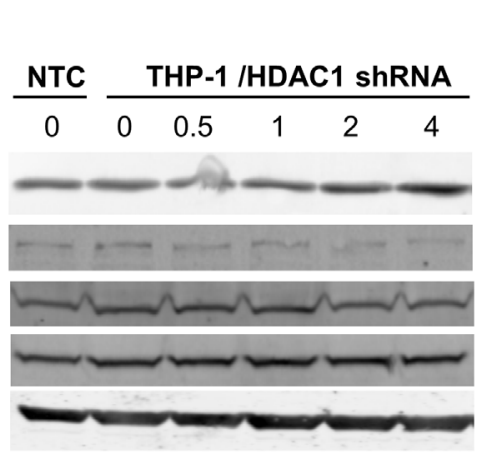

B

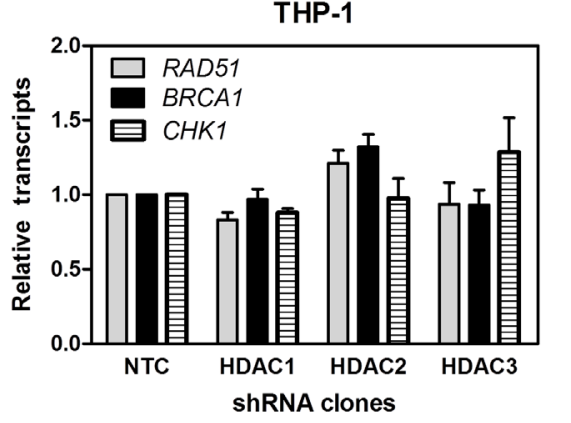

E
NTC THP-1/HDAC2 ShRNA

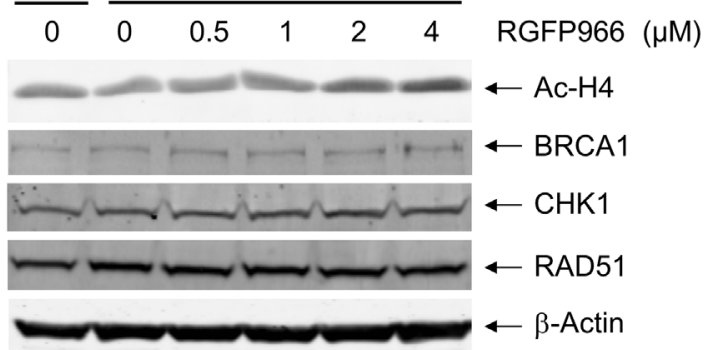

Figure 4: HDACs 1 and 2 cooperate in regulating BRCA1, CHK1, and RAD51 expression in AML cells. (A and C) THP-1 cells were infected with HDAC1 (THP-1/HDAC1), HDAC2 (THP-1/HDAC2), HDAC3 (THP-1/HDAC3), or NTC control (THP-1/NTC) shRNA lentivirus overnight, then washed and incubated for $48 \mathrm{~h}$ prior to adding puromycin to the culture medium. Whole cell lysates were subjected to Western blotting and probed with the indicated antibodies. The fold changes for the densitometry measurements, normalized to $\beta$-actin and then compared to no drug treatment control, are indicated. These Western blots were previously published [15]. (B) Total RNAs were isolated from the transfected cells and gene transcript levels were determined by Real-time RT-PCR. Transcript levels were normalized to GAPDH and relative expression levels were calculated using the comparative Ct method. (D and E) THP-1/HDAC1 and THP-1/HDAC2 cells were treated with RGFP966 for $48 \mathrm{~h}$. Whole cell lysates were subjected to Western blotting and probed with the indicated antibodies. 


\section{MATERIALS AND METHODS}

\section{Drugs}

MGCD0103, MC1568, Tubastatin A, RGFP966, and FK228 (also called Romidepsin or depsipeptide) were purchased from Selleck Chemicals (Houston, TX). Daunorubicin (DNR) and cytarabine (Ara-C) were purchased from Sigma-Aldrich (St. Louis, MO).

\section{Cell culture}

The THP-1 cell line was purchased from the American Type Culture Collection (Manassas, VA). The OCI-AML3 cell line was purchased from the German Collection of Microorganisms and Cell Cultures (DSMZ, Braunschweig, Germany). The THP-1 cell line was cultured in RPMI 1640 and the OCI-AML3 cell line was cultured in alpha-MEM with $10-15 \%$ fetal
A

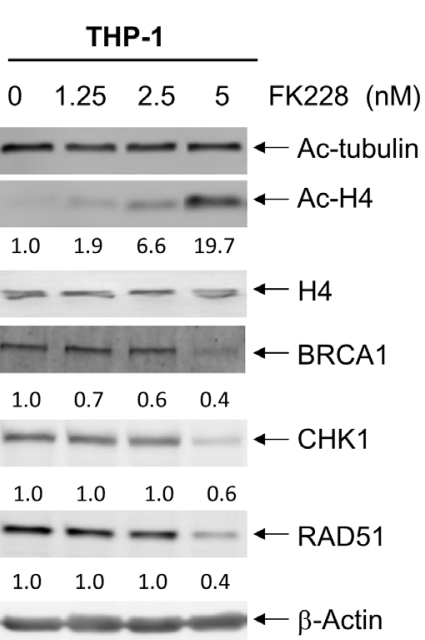

C

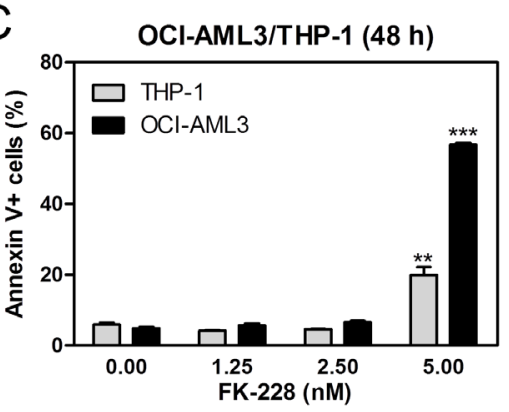

B

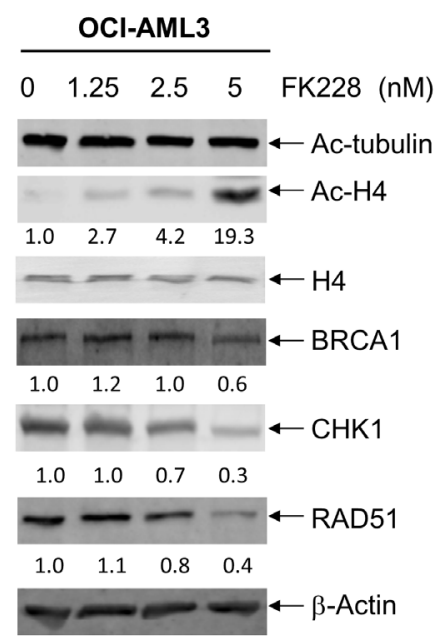

D

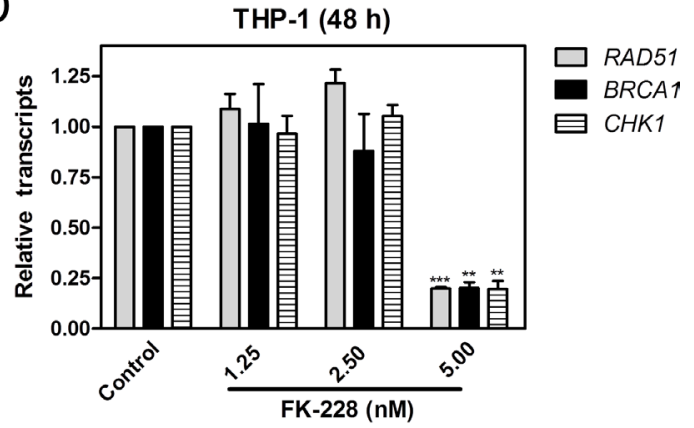

$E$

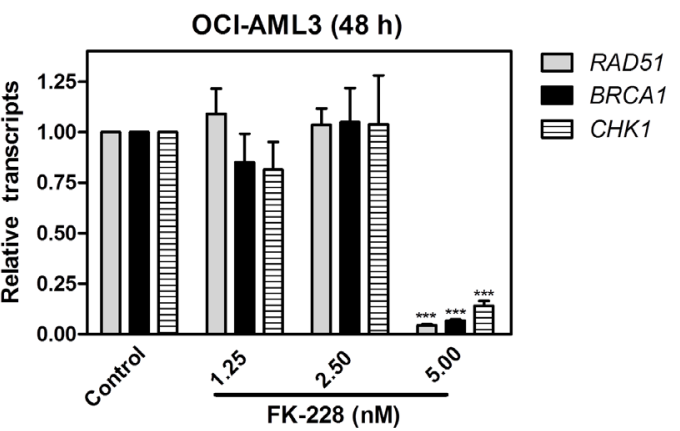

Figure 5: FK228 decreases expression of BRCA1, CHK1, and RAD51 by inhibiting HDAC1 and HDAC2 in AML cells. (A and B) THP-1 and OCI-AML3 cells were treated with FK228 for $48 \mathrm{~h}$ and whole cell lysates were subjected to Western blotting and probed with the indicated antibodies. The fold changes for the densitometry measurements, normalized to $\beta$-actin and then compared to no drug treatment control, are indicated. (C) THP-1 and OCI-AML3 cells were treated with FK228 for $48 \mathrm{~h}$ and then subjected to Annexin V-FITC/PI staining and flow cytometry analyses. ${ }^{*}$ indicates $p<0.01$ and $* * *$ indicates $p<0.001$. (D and E) THP-1 and OCI-AML3 cells were treated with FK228 for $48 \mathrm{~h}$. Total RNAs were isolated from treated cells and gene transcript levels were determined by Real-time RT-PCR. Transcript levels were normalized to GAPDH and relative expression levels were calculated using the comparative Ct method. $* *$ indicates $p<0.01$ and $* * *$ indicates $p<0.001$. 
bovine serum (Life Technologies, Grand Island, NY), $2 \mathrm{mM}$ L-glutamine, $100 \mathrm{U} / \mathrm{ml}$ penicillin and $100 \mu \mathrm{g} / \mathrm{ml}$ streptomycin. All cells were cultured in a $37^{\circ} \mathrm{C}$ humidified atmosphere containing 5\% CO2/95\% air.

\section{Enzymatic assays of class I HDACs following immunoprecipitation (IP)}

THP-1 cells were treated with variable concentrations of MGCD0103 for $48 \mathrm{~h}$ and lysed in Cell Lysis Buffer $[20 \mathrm{mmol} / \mathrm{L}$ Tris- $\mathrm{HCl}(\mathrm{pH} 8), 0.15 \mathrm{~mol} / \mathrm{L}$
$\mathrm{NaCl}, 10 \%$ glycerol, and $0.5 \% \mathrm{NP} 40$ ] on ice for 2 hours. After centrifugation $(12,000 \times \mathrm{g}$ for 15 minutes $), 500 \mu \mathrm{g}$ of the supernatant fraction (cell lysate) was incubated with $2 \mu \mathrm{g}$ rabbit IgG, anti-HDAC1, anti-HDAC3 (Bethyl Labs, Montgomery, TX), anti-HDAC2 (CycLex, Nagano, Japan) or $1000 \mu \mathrm{g}$ of supernatant fraction was incubated with $2 \mu \mathrm{g}$ anti-HDAC8 (Santa Cruz Biotechnology, California) overnight at $4^{\circ} \mathrm{C}$, followed by incubation with $30 \mu 1$ of Protein A/G Dynabeads (Life Technologies) for 3 hours at $4^{\circ} \mathrm{C}$. The beads were washed three times with ice cold PBS and resuspended in HDAC Assay Buffer
A

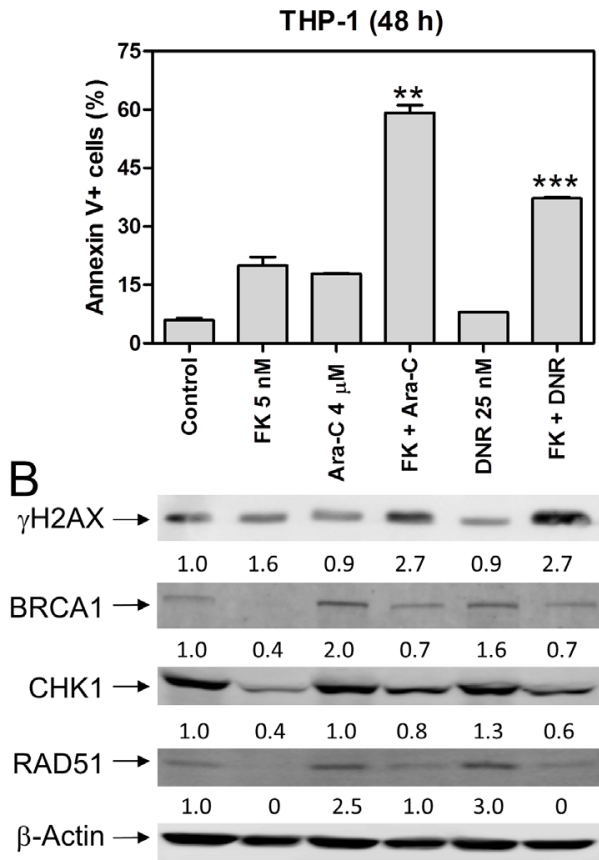

$E$

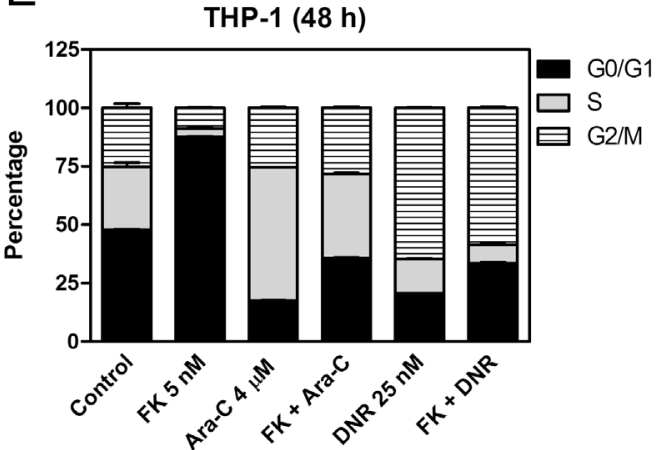

C

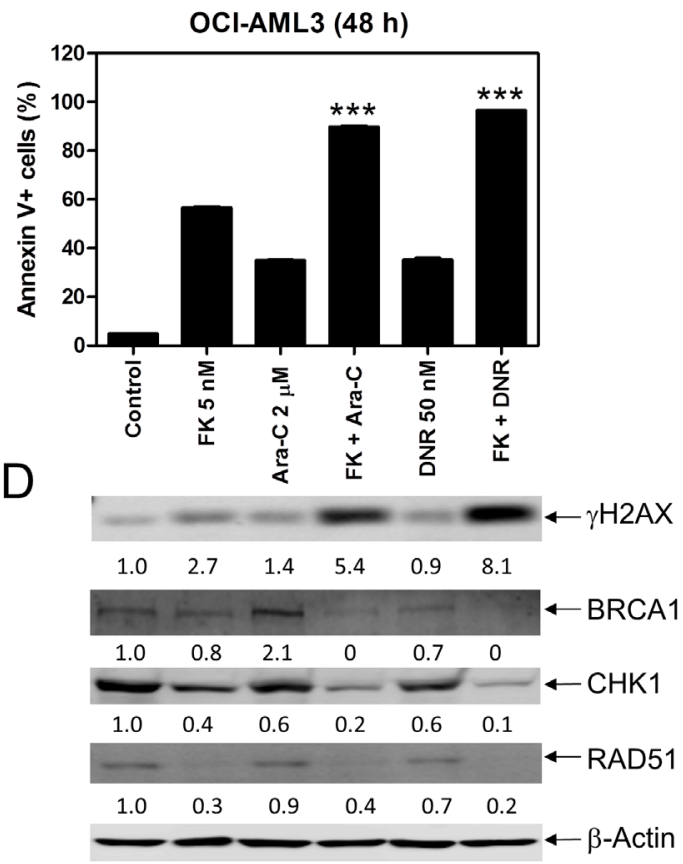

$\mathrm{F}$

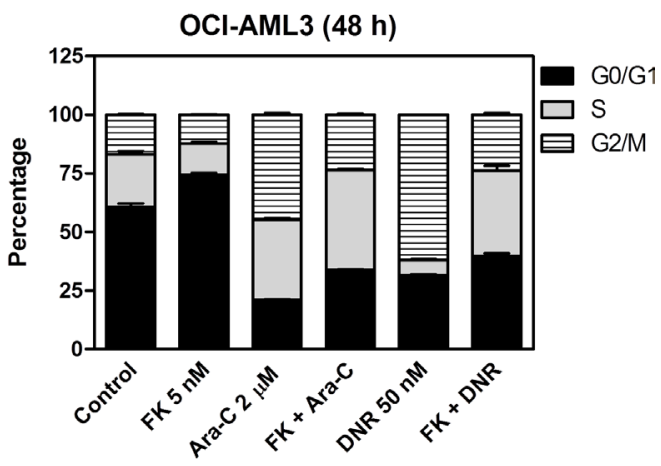

Figure 6: FK228 cooperates with ara-C or DNR in inducing apoptosis and abrogates $\mathrm{S}$ and/or G2/M cell cycle checkpoint activation induced by ara-C or DNR in THP-1 and OCI-AML3 AML cells. (A) THP-1 cells were treated with FK228 and ara-C or DNR, alone or in combination, for $48 \mathrm{~h}$ and then subjected to Annexin V-FITC/PI staining and flow cytometry analyses. **indicates $p<0.01$ and ${ }^{* * *}$ indicates $p<0.001$. (B) Whole cell lysates were subjected to Western blotting and probed with the indicated antibodies. The fold changes for the densitometry measurements, normalized to $\beta$-actin and then compared to no drug treatment control, are indicated. (C) OCI-AML3 cells were treated with FK228 and ara-C or DNR, alone or in combination, for $48 \mathrm{~h}$ and then subjected to Annexin V-FITC/PI staining and flow cytometry analyses. ${ }^{* * *}$ indicates $p<0.001$. (D) Whole cell lysates were subjected to Western blotting and probed with the indicated antibodies. The fold changes for the densitometry measurements, normalized to $\beta$-actin and then compared to no drug treatment control, are indicated. (E and F) THP-1 and OCI-AML3 cells were treated with FK228 and ara-C or DNR, alone or in combination for $48 \mathrm{~h}$ and then fixed with ethanol and stained with PI for cell cycle analysis. 
[40 mL; 20 mmol/L Tris- $\mathrm{HCl}$ (pH 8), $125 \mathrm{mmol} / \mathrm{L} \mathrm{NaCl}$, and $1 \%$ glycerol] and then HDAC enzymatic activities were measured using the CycLexH HDACs Deacetylase Fluorometric Assay kit (CycLex), or heated at $95^{\circ} \mathrm{C}$ for 5 min in $30 \mu \mathrm{l}$ loading buffer for Western blotting.

\section{Quantification of gene expression by real-time RT-PCR}

Total RNA was extracted using TRIzol (Life Technologies), cDNAs were prepared from $2 \mu \mathrm{g}$ total RNA using random hexamer primers and a RT-PCR kit (Life Technologies), and purified using the QIAquick PCR Purification Kit (Qiagen, Valencia, CA), as previously described [23, 24]. Transcripts for $B R C A 1, C H K 1$, and $R A D 51$ were quantitated using TaqMan probes (Life Technologies) and a LightCycler ${ }^{\circledR} 480$ real-time PCR machine (Roche Diagnostics, Indianapolis, IN), based on the manufacturer's instructions. Real-time PCR results are expressed as mean values from 3 independent experiments using the same cDNA preparations and were normalized to GAPDH. Fold changes were calculated using the comparative Ct method [25].

\section{Western blot analysis}

Cells were lysed in the presence of protease and phosphatase inhibitors (Roche Diagnostics). Whole cell lysates were subjected to SDS-polyacrylamide gel electrophoresis, electrophoretically transferred onto polyvinylidene difluoride (PVDF) membranes (Thermo Fisher Inc., Rockford, IL), and immunoblotted with anti-acetyl-histone 4 (ac-H4), -H4, -acetyl-tubulin (ac-tubulin, Upstate Biotechnology, Lake Placid, NY), $-\gamma \mathrm{H} 2 \mathrm{AX},-\mathrm{HDAC} 1,-\mathrm{HDAC} 2,-\mathrm{HDAC} 3$ (Cell Signaling Technology), -BRCA1, -RAD51, -CHK1 (Santa Cruz Biotechnology) or $-\beta$-actin (Sigma, St Louis, MO) antibody, as previously described [26, 27]. Immunoreactive proteins were visualized using the Odyssey Infrared Imaging System (Li-Cor, Lincoln, NE, USA).

\section{Apoptosis}

AML cells were treated with the indicated drugs for $48 \mathrm{~h}$ and then subjected to flow cytometry analysis to determine drug-induced apoptosis using an Annexin V-fluorescein isothiocyanate (FITC)/PI apoptosis Kit (Beckman Coulter; Brea, CA, USA), as previously described [24, 28]. Experiments were performed 3 independent times in triplicates. Results from one representative experiment are shown.

\section{Cell cycle progression}

Cells were treated with the indicated drugs for $48 \mathrm{~h}$. The cells were harvested and fixed with ice-cold $80 \%$ (v/v) ethanol for $24 \mathrm{~h}$. The cells were pelleted, washed with PBS, and resuspended in PBS containing $50 \mu \mathrm{g} / \mathrm{mL}$ PI, $0.1 \%$ Triton X-100 (v/v), and $1 \mu \mathrm{g} / \mathrm{mL}$ DNase-free RNase. DNA content was determined by flow cytometry analysis using a FACS Calibur flow cytometer (Becton Dickinson), as previously described [14]. Cell cycle analysis was performed using ModFit LT 3.0 (Becton Dickinson).

\section{shRNA knockdown of $H D A C 1, H D A C 2$, and HDAC3 in THP-1 cells}

HDAC1, HDAC2, HDAC3, and non-target control (NTC) shRNA lentiviral particles were purchased from Sigma-Aldrich and used to infect THP-1 cells. THP-1 cells were transduced overnight and then cultured for an additional $48 \mathrm{~h}$ prior to selection with puromycin. A pool of infected cells was expanded and tested for HDAC1, HDAC2, and HDAC3 expression by Western blotting. A pool of cells from the NTC shRNA lentiviral transduction was used as the negative control.

\section{Statistical analysis}

Differences in cell apoptosis and $B R C A 1, C H K 1$, and $R A D 51$ transcript levels between treated (individually or combined) and untreated cells were compared using the paired t-test. Statistical analyses were performed with GraphPad Prism 5.0. Error bars represent \pm SEM. The level of significance was set at $p<0.05$.

\section{ACKNOWLEDGMENTS}

This study was supported by Jilin University, Changchun, China, China Scholarship Council, the Barbara Ann Karmanos Cancer Institute, Wayne State School of Medicine, and the Children's Hospital of Michigan Foundation.

\section{CONFLICTS OF INTEREST}

The authors declare no competing financial interests.

\section{GRANT SUPPORT}

This study was supported by grants from the National Natural Science Foundation of China, NSFC 31271477 and NSFC 31471295, China Scholarship Council, Hyundai Hope on Wheels, LaFontaine Family/U Can-Cer Vive Foundation, Kids Without Cancer, Children's Hospital of Michigan Foundation, Decerchio/Guisewite Family, Justin's Gift, Elana Fund, Ginopolis/Karmanos Endowment and the Ring Screw Textron Endowed Chair for Pediatric Cancer Research. The funders had no role in study design, data collection, analysis and interpretation of data, decision to publish, or preparation of the manuscript. 


\section{REFERENCES}

1. Quintas-Cardama A, Santos FP, Garcia-Manero G. Histone deacetylase inhibitors for the treatment of myelodysplastic syndrome and acute myeloid leukemia. Leukemia. 2011; 25:226-235.

2. Burnett A, Wetzler M, Lowenberg B. Therapeutic advances in acute myeloid leukemia. J Clin Oncol. 2011; 29:487-494.

3. Pollyea DA, Kohrt HE, Medeiros BC. Acute myeloid leukaemia in the elderly: a review. Br J Haematol. 2011; 152:524-542.

4. Bolden JE, Peart MJ, Johnstone RW. Anticancer activities of histone deacetylase inhibitors. Nat Rev Drug Discov. 2006; 5:769-784.

5. Wagner JM, Hackanson B, Lubbert M, Jung M. Histone deacetylase (HDAC) inhibitors in recent clinical trials for cancer therapy. Clin Epigenetics. 2010; 1:117-136.

6. Garcia-Manero G, Assouline S, Cortes J, Estrov Z, Kantarjian H, Yang H, Newsome WM, Miller WH, Jr., Rousseau C, Kalita A, Bonfils C, Dubay M, et al. Phase 1 study of the oral isotype specific histone deacetylase inhibitor MGCD0103 in leukemia. Blood. 2008; 112:981-989.

7. Garcia-Manero G, Yang H, Bueso-Ramos C, Ferrajoli A, Cortes J, Wierda WG, Faderl S, Koller C, Morris G, Rosner G, Loboda A, Fantin VR, Randolph SS, et al. Phase 1 study of the histone deacetylase inhibitor vorinostat (suberoylanilide hydroxamic acid [SAHA]) in patients with advanced leukemias and myelodysplastic syndromes. Blood. 2008; 111:1060-1066.

8. Kuendgen A, Schmid M, Schlenk R, Knipp S, Hildebrandt B, Steidl C, Germing U, Haas R, Dohner H, Gattermann N. The histone deacetylase (HDAC) inhibitor valproic acid as monotherapy or in combination with alltrans retinoic acid in patients with acute myeloid leukemia. Cancer. 2006; 106:112-119.

9. Schaefer EW, Loaiza-Bonilla A, Juckett M, DiPersio JF, Roy V, Slack J, Wu W, Laumann K, Espinoza-Delgado I, Gore SD. A phase 2 study of vorinostat in acute myeloid leukemia. Haematologica. 2009; 94:1375-1382.

10. Byrd JC, Marcucci G, Parthun MR, Xiao JJ, Klisovic RB, Moran M, Lin TS, Liu S, Sklenar AR, Davis ME, Lucas DM, Fischer B, Shank R, et al. A phase 1 and pharmacodynamic study of depsipeptide (FK228) in chronic lymphocytic leukemia and acute myeloid leukemia. Blood. 2005; 105:959-967.

11. Nolan L, Johnson PW, Ganesan A, Packham G, Crabb SJ. Will histone deacetylase inhibitors require combination with other agents to fulfil their therapeutic potential? Br J Cancer. 2008; 99:689-694.

12. Gojo I, Tan M, Fang HB, Sadowska M, Lapidus R, Baer MR, Carrier F, Beumer JH, Anyang BN, Srivastava RK, Espinoza-Delgado I, Ross DD. Translational phase I trial of vorinostat (suberoylanilide hydroxamic acid) combined with cytarabine and etoposide in patients with relapsed, refractory, or high-risk acute myeloid leukemia. Clinical cancer research Clin Cancer Res. 2013; 19:1838-1851.

13. Garcia-Manero G, Tambaro FP, Bekele NB, Yang H, Ravandi F, Jabbour E, Borthakur G, Kadia TM, Konopleva MY, Faderl S, Cortes JE, Brandt M, Hu Y, et al. Phase II trial of vorinostat with idarubicin and cytarabine for patients with newly diagnosed acute myelogenous leukemia or myelodysplastic syndrome. J Clin Oncol. 2012; 30:2204-2210.

14. Xie C, Drenberg C, Edwards H, Caldwell JT, Chen W, Inaba H, Xu X, Buck SA, Taub JW, Baker SD, Ge Y. Panobinostat enhances cytarabine and daunorubicin sensitivities in AML cells through suppressing the expression of BRCA1, CHK1, and Rad51. PloS one. 2013; 8:e79106.

15. Xu X, Xie C, Edwards H, Zhou H, Buck SA, Ge Y. Inhibition of histone deacetylases 1 and 6 enhances cytarabine-induced apoptosis in pediatric acute myeloid leukemia cells. PloS one. 2011; 6:e17138.

16. Redon CE, Nakamura AJ, Zhang YW, Ji JJ, Bonner WM, Kinders RJ, Parchment RE, Doroshow JH, Pommier Y. Histone gammaH2AX and poly(ADP-ribose) as clinical pharmacodynamic biomarkers. Clin Cancer Res. 2010; 16:4532-4542.

17. Miller KM, Tjeertes JV, Coates J, Legube G, Polo SE, Britton S, Jackson SP. Human HDAC1 and HDAC2 function in the DNA-damage response to promote DNA nonhomologous end-joining. Nat Struct Mol Biol. 2010; 17:1144-1151.

18. Krumm A, Barckhausen C, Kucuk P, Tomaszowski KH, Loquai C, Fahrer J, Kramer OH, Kaina B, Roos WP. Enhanced Histone Deacetylase Activity in Malignant Melanoma Provokes RAD51 and FANCD2-Triggered Drug Resistance. Cancer Res. 2016; 76:3067-3077.

19. Thurn KT, Thomas S, Raha P, Qureshi I, Munster PN. Histone deacetylase regulation of ATM-mediated DNA damage signaling. Mol Cancer Ther. 2013; 12:2078-2087.

20. Kachhap SK, Rosmus N, Collis SJ, Kortenhorst MS, Wissing MD, Hedayati M, Shabbeer S, Mendonca J, Deangelis J, Marchionni L, Lin J, Hoti N, Nortier JW, et al. Downregulation of homologous recombination DNA repair genes by HDAC inhibition in prostate cancer is mediated through the E2F1 transcription factor. PloS one. 2010; 5:e11208.

21. Brazelle W, Kreahling JM, Gemmer J, Ma Y, Cress WD, Haura E, Altiok S. Histone deacetylase inhibitors downregulate checkpoint kinase 1 expression to induce cell death in non-small cell lung cancer cells. PloS one. 2010; 5:e14335.

22. Lai TH, Ewald B, Zecevic A, Liu C, Sulda M, Papaioannou D, Garzon R, Blachly JS, Plunkett W, Sampath D. HDAC Inhibition Induces MicroRNA-182, which Targets RAD51 and Impairs HR Repair to Sensitize Cells to Sapacitabine in Acute Myelogenous Leukemia. Clin Cancer Res. 2016; 22:3537-3549. 
23. Niu X, Wang G, Wang Y, Caldwell JT, Edwards H, Xie C, Taub JW, Li C, Lin H, Ge Y. Acute myeloid leukemia cells harboring MLL fusion genes or with the acute promyelocytic leukemia phenotype are sensitive to the Bcl-2-selective inhibitor ABT-199. Leukemia. 2014; 28:1557-1560.

24. Edwards H, Xie C, LaFiura KM, Dombkowski AA, Buck SA, Boerner JL, Taub JW, Matherly LH, Ge Y. RUNX1 regulates phosphoinositide 3-kinase/AKT pathway: role in chemotherapy sensitivity in acute megakaryocytic leukemia. Blood. 2009; 114:2744-2752.

25. Livak KJ, Schmittgen TD. Analysis of relative gene expression data using real-time quantitative PCR and the 2(-Delta Delta C(T)) Method. Methods. 2001; 25:402-408.

26. Ge Y, Dombkowski AA, LaFiura KM, Tatman D, Yedidi RS, Stout ML, Buck SA, Massey G, Becton DL, Weinstein HJ,
Ravindranath Y, Matherly LH, Taub JW. Differential gene expression, GATA1 target genes, and the chemotherapy sensitivity of Down syndrome megakaryocytic leukemia. Blood. 2006; 107:1570-1581.

27. Ge Y, Stout ML, Tatman DA, Jensen TL, Buck S, Thomas RL, Ravindranath Y, Matherly LH, Taub JW. GATA1, cytidine deaminase, and the high cure rate of Down syndrome children with acute megakaryocytic leukemia. J Natl Cancer Inst. 2005; 97:226-231.

28. Xie C, Edwards H, Xu X, Zhou H, Buck SA, Stout ML, Yu Q, Rubnitz JE, Matherly LH, Taub JW, Ge Y. Mechanisms of synergistic antileukemic interactions between valproic acid and cytarabine in pediatric acute myeloid leukemia. Clin Cancer Res. 2010; 16:5499-5510. 